\title{
Bioavailability, Biotransformation, and Excretion of the Covalent Bruton Tyrosine Kinase Inhibitor Acalabrutinib in Rats, Dogs, and Humans ${ }^{\mathbb{S}}$
}

\author{
Terry Podoll, Paul G. Pearson, Jerry Evarts, Tim Ingallinera, Elena Bibikova, Hao Sun, \\ Mark Gohdes, Kristen Cardinal, Mitesh Sanghvi, and J. Greg Slatter
}

Acerta Pharma, South San Francisco, California (T.P., J.E., T.I., E.B., J.G.S.); Pearson Pharma Partners, Westlake Village, California (P.G.P.); Covance, Madison, Wisconsin (H.S., M.G., K.C.); and Xceleron, Germantown, Maryland (M.S.)

Received September 12, 2018; accepted November 7, 2018

\section{ABSTRACT}

Acalabrutinib is a targeted, covalent inhibitor of Bruton tyrosine kinase (BTK) with a unique 2-butynamide warhead that has relatively lower reactivity than other marketed acrylamide covalent inhibitors. A human $\left[{ }^{14} \mathrm{C}\right]$ microtracer bioavailability study in healthy subjects revealed moderate intravenous clearance $(39.4 \mathrm{l} / \mathrm{h})$ and an absolute bioavailability of $25.3 \% \pm 14.3 \%(n=8)$. Absorption and elimination of acalabrutinib after a $100 \mathrm{mg}\left[{ }^{14} \mathrm{C}\right]$ microtracer acalabrutinib oral dose was rapid, with the maximum concentration reached in $<1$ hour and elimination half-life values of $<2$ hours. Low concentrations of radioactivity persisted longer in the blood cell fraction and a peripheral blood mononuclear cell subfraction (enriched in target BTK) relative to plasma. $\left[{ }^{14} \mathrm{C}\right]$ Acalabrutinib was metabolized to more than three dozen metabolites detectable by liquid chromatography-tandem mass spectrometry, with primary metabolism by CYP3A-mediated oxidation of the pyrrolidine ring, thiol conjugation of the butynamide warhead, and amide hydrolysis. A major active, circulating, pyrrolidine ring-opened metabolite, ACP-5862 (4[8-amino-3-[4-(but-2-ynoylamino)butanoyl]imidazo[1,5-a]pyrazin-1yl]-N-(2-pyridyl)benzamide), was produced by CYP3A oxidation. Novel enol thioethers from the 2-butynamide warhead arose from glutathione and/or cysteine Michael additions and were subject to hydrolysis to a $\beta$-ketoamide. Total radioactivity recovery was $95.7 \% \pm 4.6 \%(n=6)$, with $12.0 \%$ of dose in urine and $83.5 \%$ in feces. Excretion and metabolism characteristics were generally similar in rats and dogs. Acalabrutinib's highly selective, covalent mechanism of action, coupled with rapid absorption and elimination, enables high and sustained BTK target occupancy after twice-daily administration.

\section{Introduction}

Acalabrutinib (Calquence; AstraZeneca, Cambridge, UK) is a highly selective, potent, orally administered, targeted covalent inhibitor (TCI) of Bruton tyrosine kinase (BTK) and received accelerated approval from the U.S. Food and Drug Administration (FDA) in October 2017 for treatment of relapsed/refractory mantle cell lymphoma (Wang et al., 2018). BTK is a Tec family kinase expressed in B cells, myeloid cells, mast cells, and platelets and plays an integral role in B-cell receptor signaling that is responsible for the proliferation and survival of malignant B cells (Buggy and Elias, 2012). Irreversible inactivation of

This work was supported by Acerta Pharma.

https://doi.org/10.1124/dmd.118.084459.

S This article has supplemental material available at dmd.aspetjournals.org.
BTK was established as a valuable clinical target for the treatment of B-cell malignancies by the first-in-class agent, ibrutinib (Ponader and Burger, 2014). Adverse effects of ibrutinib therapy include bleeding and atrial fibrillation, which were postulated to involve off-target activity against other Tec (Wang et al., 2018) and Src (Barf et al., 2017) family kinases. The discovery and development of acalabrutinib focused on reducing overall warhead reactivity and improving selectivity for BTK (Barf et al., 2017).

Recent regulatory approvals of TCIs of other protein kinases have led to interest in identifying desirable physicochemical and absorption, distribution, metabolism, and excretion (ADME) properties in drug candidates (Liu et al., 2013; Moghaddam et al., 2014; Baillie, 2016; Lonsdale and Ward, 2018). Unlike drugs that interact reversibly with their targets and generally require sustained systemic exposure to achieve target coverage over the dose interval, potent TCIs can drive

ABBREVIATIONS: ACP-5862, 4-[8-amino-3-[4-(but-2-ynoylamino)butanoyl]imidazo[1,5-a]pyrazin-1-yl]-N-(2-pyridyl)benzamide; ACP-5197, 4-[8-amino-3[(2S)-1-but-2-ynoylpyrrolidin-2-yl]imidazo[1,5-a]pyrazin-1-yl]benzoic acid; ACP-5530, (2S)-2-amino-5-[(1R)-1-[(E)-3-[(2S)-2-[8-amino-1-[4-(2-pyridylcarbamoyl) phenyl]imidazo[1,5-a]pyrazin-3-yl]pyrrolidin-1-yl]-1-methyl-3-oxo-prop-1-enyl]sulfanylmethyl]-2-(carboxymethylamino)-2-oxo-ethyl]amino]-5-oxo-pentanoic acid; ACP-5461, (2R)-2-amino-3-[(E)-3-[(2S)-2-[8-amino-1-[4-(2-pyridylcarbamoyl)phenyl]imidazo[1,5-a]pyrazin-3-yl]pyrrolidin-1-yl]-1-methyl-3-oxo-prop-1enyl]sulfanyl-propanoic acid; ACP-5134, 4-[8-amino-3-[(2S)-1-(3-oxobutanoyl)pyrrolidin-2-yl]imidazo[1,5-a]pyrazin-1-yl]-N-(2-pyridyl)benzamide; ACP5531, 2-[(2R)-2-amino-3-[(E)-3-[(2S)-2-[8-amino-1-[4-(2-pyridylcarbamoyl)phenyl]imidazo[1,5-a]pyrazin-3-yl]pyrrolidin-1-yl]-1-methyl-3-oxo-prop-1-enyl] sulfanyl-propanoyl]amino]acetic acid; ADME, absorption, distribution, metabolism, and excretion; AMS, accelerator mass spectrometry; AUC, area under the plasma concentration-time curve; BCRP, breast cancer resistance protein; BTK, Bruton tyrosine kinase; CL, clearance; CV, coefficient of variation; FDA, U.S. Food and Drug Administration; GSH, glutathione; GST, glutathione S-transferase; HER, human epidermal growth factor receptor; HPLC, highperformance liquid chromatography; LC, liquid chromatography; LSC, liquid scintillation counting; MS, mass spectrometry; MS/MS, tandem mass spectrometry; PBMC, peripheral blood mononuclear cell; PK, pharmacokinetics; $t_{1 / 2}$, half-life; TCl, targeted covalent inhibitor; $T_{\text {max }}$, time of maximum observed plasma concentration. 
high target occupancy after a relatively brief systemic exposure. This can result in prolonged pharmacodynamic effects, with the duration of effect related to the time required for target resynthesis (Singh et al., 2011; Barf and Kaptein, 2012).

Prior to acalabrutinib, clinically approved protein kinase TCIs included ibrutinib (BTK), osimertinib (epidermal growth factor receptor), neratinib (human epidermal growth factor receptor HER2), and afatinib (HER1, HER2, and HER4). Although they target distinct protein kinases, all four of these agents employ an electrophilic acrylamide warhead that targets a selected cysteine thiol nucleophile in the ATP binding pocket of each protein kinase. The acrylamide functionality has been used more than any other electrophilic warhead in the development of TCIs of protein kinases (Zhao and Bourne, 2018). They have the advantage of high potency against their respective protein kinase targets; however, they can lack kinase selectivity and result in off-target binding to plasma proteins (Chandrasekaran et al., 2010; Stopfer et al., 2012; Scheers et al., 2015; Dickinson et al., 2016). Osimertinib covalent plasma protein binding is extensive enough to suggest that it provides a significant route of clearance (Dickinson et al., 2016). Glutathione (GSH) conjugation of the acrylamide warhead is a metabolic route of approved TCIs (Shibata and Chiba, 2015).

Acalabrutinib contains a 2-butynamide electrophilic warhead that is less reactive than an analog with the corresponding acrylamide warhead (Barf et al., 2017). The cysteine- 481 target nucleophile in BTK has a proximal asparagine residue that lowers the $\mathrm{p} K_{\mathrm{a}}$ of the cysteine- 481 thiol, thereby increasing its nucleophilicity. This results in improved selectivity (Barf et al., 2017), and the metabolic fate of this functional group has not been previously described.

Initial clinical studies indicate that acalabrutinib has optimal characteristics of a TCI-namely, rapid absorption, low systemic exposure, and a short plasma half-life $\left(t_{1 / 2}\right)$. This results in high and sustained selective BTK occupancy and durable clinical effectiveness with twicedaily dosing (Byrd et al., 2016; Barf et al., 2017; Wang et al., 2018). The ADME properties of $\left[{ }^{14} \mathrm{C}\right]$ acalabrutinib in rats, dogs, and humans are reported here.

\section{Materials and Methods}

\section{Radiolabeled Acalabrutinib and Reference Standards}

$\left[{ }^{14} \mathrm{C}\right]$ Acalabrutinib (1,4-[8-amino-3-[(2S)-1-but-2-ynoylpyrrolidin-2-yl]imidazo[1,5$\alpha$ ]pyrazin-1-yl]- $N$-(2-pyridyl)-benzamide) was labeled in the carbonyl carbon atom of the 2-pyridylbenzamide moiety (Fig. 1), with specific activity of $57 \mathrm{mCi} / \mathrm{mmol}$. Metabolite standards were prepared by Kalexsyn (Kalamazoo, MI) or Acerta Pharma BV (Oss, Netherlands; data on file).

\section{Reversible Plasma Protein Binding and Red Blood Cell Partitioning}

Prior to conducting plasma incubations, nonspecific binding to the low-binding polycarbonate tubes (Sumitomo Bakelite, Akita, Japan) by both acalabrutinib and ACP-5862 (4-[8-amino-3-[4-(but-2-ynoylamino)butanoyl]imidazo[1,5-a]pyrazin1-yl]-N-(2-pyridyl)benzamide) was determined in phosphate-buffered saline using liquid chromatography (LC)-tandem mass spectrometry (MS/MS). In addition, the stability of acalabrutinib $(1 \mu \mathrm{M})$ was established in potassium oxalate/sodium fluoride male mouse and rat plasma, EDTA male dog and monkey plasma, and lithium heparin male human plasma for 16 hours at $4{ }^{\circ} \mathrm{C}$ using low-binding

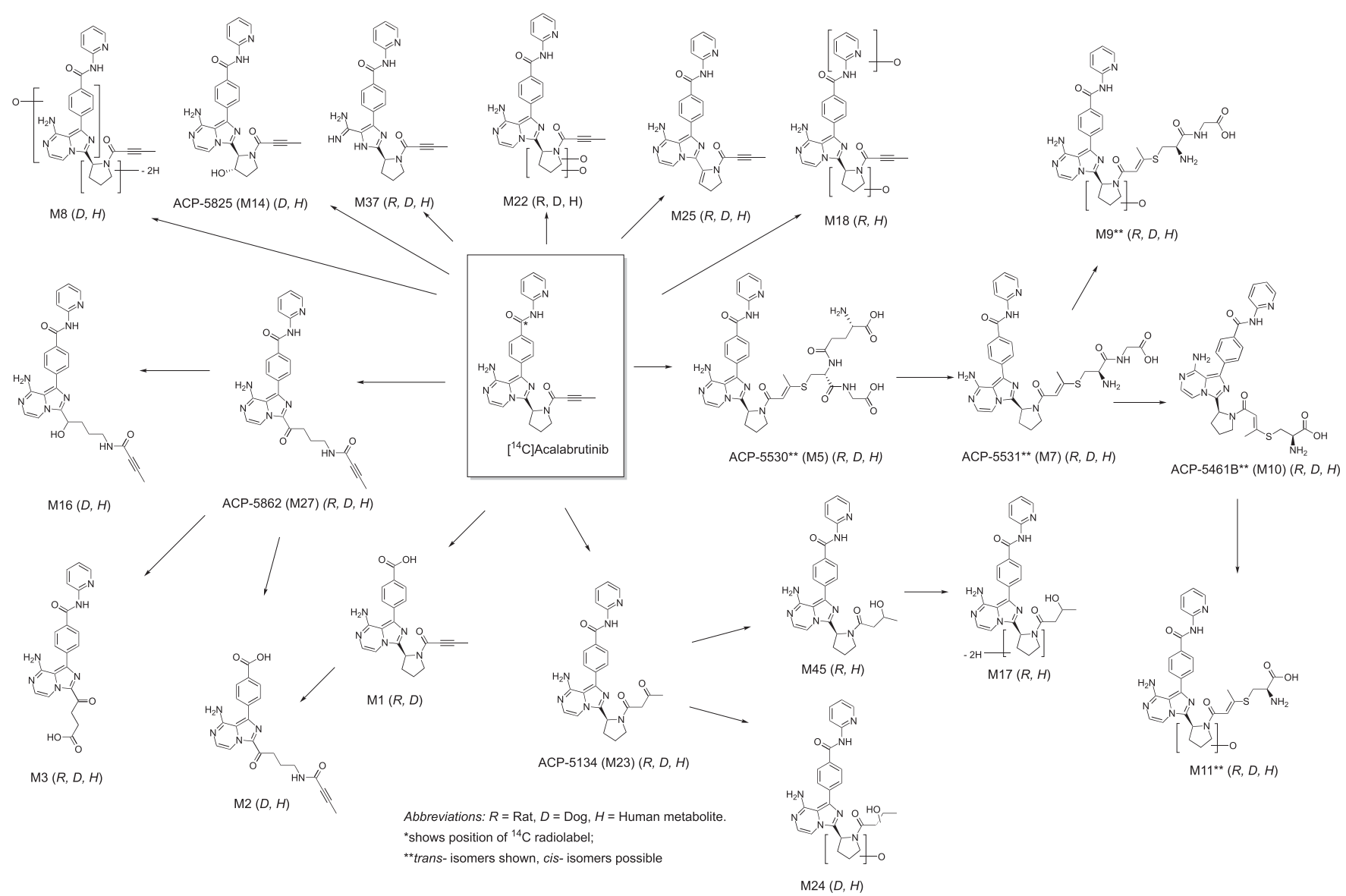

Fig. 1. Proposed metabolic pathways of acalabrutinib. 
polycarbonate tubes and LC-MS/MS analysis. Then the free fraction of $\left[{ }^{14} \mathrm{C}\right]$ acalabrutinib $(1,3$, and $10 \mu \mathrm{M})$ in plasma was determined by ultracentrifugation with liquid scintillation counting (LSC) of the supernatant. $\left[{ }^{14} \mathrm{C}\right]$ Acalabrutinib reversible binding to pure human serum albumin solution (physiologic, $40 \mathrm{mg} / \mathrm{ml}$ ) and $\alpha 1$-acid glycoprotein solution (physiologic, $20 \mu \mathrm{M}$ ) was determined similarly. The free fraction of the metabolite ACP-5862 $(1$ and $10 \mu \mathrm{M})$ was determined similarly by ultracentrifugation and LC-MS/MS quantification. Red blood cell partitioning of acalabrutinib and ACP-5862 was determined after a 15 -minute incubation of male whole blood at $37^{\circ} \mathrm{C}$ and centrifugation, followed by LC-MS/MS of plasma. The radiochemical purity of $\left[{ }^{14} \mathrm{C}\right]$ acalabrutinib was $96.7 \%$ 20 days before the protein binding experiments and $96.6 \% 23$ days after using radiochromatographic analysis.

\section{ADME of $\left[{ }^{14} \mathrm{C}\right]$ Acalabrutinib in Rats and Dogs}

The pharmacokinetics (PK) and ADME of $\left[{ }^{14} \mathrm{C}\right]$ acalabrutinib were characterized in male and female Sprague-Dawley rats (Hsd:Sprague Dawley SD; Envigo RMS Inc., Indianapolis, IN) aged 7 to 8 weeks at dosing ( $n=15 / \mathrm{sex}$ for PK, $n=3 /$ sex for excreta and carcass $)$ and Beagle dogs $(n=3 / \mathrm{sex}$; Covance Research Products, Cumberland, VA) that had been assigned to groups and after oral administration of a 100 and $30 \mathrm{mg} / \mathrm{kg}$ dose of $\left[{ }^{14} \mathrm{C}\right]$ acalabrutinib (50 and $10 \mu \mathrm{Ci} / \mathrm{kg}$ ), respectively. Whole blood, plasma, urine, and feces samples were collected at appropriate intervals through 96 hours postdose. Plasma samples were extracted twice and feces homogenates were extracted three times into methanol (sample/methanol, 1:3 v/v). For each sample, the mixture was sonicated (10 minutes), vortexed (10 minutes), and centrifuged (2400g, 10 minutes at ambient temperature) and the supernatant was separated. After the second or third extraction, the combined supernatants were reduced to dryness under a stream of nitrogen at $60^{\circ} \mathrm{C}$. The extract was then reconstituted in water/methanol $(70: 30 \mathrm{v} / \mathrm{v}$, $300 \mu$ l) before analysis by LC-mass spectrometry (MS) and/or LC-MS/MS, with eluent fraction collection and TopCount (PerkinElmer, Waltham, MA) radioanalysis. Radiochromatographic profiles were used to identify and quantify metabolites. Whole blood and feces samples were processed by digestion and combustion, respectively, before they were analyzed for total radioactivity by LSC. All preclinical work was conducted at Covance Laboratories, Madison, WI, a facility accredited by the Association for Assessment and Accreditation of Laboratory Animal Care International, in compliance with applicable animal welfare regulations and after protocol approval by its Institutional Animal Care and Use Committee.

\section{Human $\left[{ }^{14} \mathrm{C}\right]$ Acalabrutinib Microtracer Absolute Bioavailability, Metabolism, and Excretion Study}

A phase I, open-label, single-center, nonrandomized two-cohort study was conducted in healthy subjects. A radiolabel microtracer dose was used to determine the absolute bioavailability of acalabrutinib in participants enrolled in cohort 1 and to determine the mass balance for those in cohort 2 . The study was performed in accordance with the ethical principles stated in the Declaration of Helsinki, FDA Code of Federal Regulations Title 21 (Parts 50, 56, 312, and 361.1), and International Conference on Harmonization guidelines for good clinical practice. All subjects provided informed consent on an institutional review board-approved protocol. Healthy male and female subjects aged 18-65 years were recruited at a single site (Covance Clinical Research Unit, Madison, WI). The use of prescribed or nonprescribed concomitant medications was not permitted in the 14 or 7 days, respectively, before the first administration of acalabrutinib unless deemed acceptable by the investigator. In addition, use of any drugs known to be significant inducers or inhibitors of cytochrome P450 enzymes and/or P-glycoprotein, including St. John's wort, was not permitted for 28 days before the administration of acalabrutinib and throughout the study. Subjects were also excluded if they had consumed alcohol-, grapefruit-, or caffeine-containing foods and beverages within 72 hours of the first administration of acalabrutinib. All subjects were screened within 28 days before study entry and could only participate in one cohort. Eligible subjects were admitted to the study center on day -1 . Subjects fasted overnight (at least 8 hours) before administration of acalabrutinib on day 1 .

Cohort 1: Absolute Bioavailability. The bioavailability of a single 100-mg dose of the oral to-be-marketed capsule formulation of unlabeled acalabrutinib was measured in male and female healthy subjects $(n=8)$ relative to a microtracer dose of $\left[{ }^{14} \mathrm{C}\right]$ acalabrutinib $(<10 \mu \mathrm{g} ; \leq 1 \mu \mathrm{Ci})$ administered as a 2-minute intravenous push ( $5 \mathrm{ml}$ of an approximately $0.194 \mu \mathrm{Ci} / \mathrm{ml}$ saline solution), timed to finish 1 hour after the unlabeled oral dose, approximating the maximum observed plasma concentration $\left(C_{\max }\right)$ reported in prior studies. Blood samples were collected at the following time points: predose; at 15, 30, 45, and 58 minutes (immediately before intravenous push); at 1 hour (immediately after the end of intravenous push), 1 hour 5 minutes, 1 hour 10 minutes, 1 hour 15 minutes, 1 hour 20 minutes, and 1 hour 30 minutes; and at 2, 3, 4, 5, 6, 8, 12, 24, 36, 48, 60, 72, and 96 hours after the oral dose. Complete urine collections were obtained at the following intervals: -12 to 0 (before dose, up to the last void within 20 minutes before oral dosing), $0-6,6-12,12-24,24-48$, and 48-72 hours after the oral dose. Plasma and urine samples were analyzed for total acalabrutinib by LC-MS/MS, and $\left[{ }^{14} \mathrm{C}\right]$ acalabrutinib was analyzed by high-performance liquid chromatography (HPLC) fractionation and accelerator mass spectrometry (AMS). The subjects were released from the clinical site after 4 days of continuous residence. The intravenous dose ${ }^{14} \mathrm{C}$ content for microtracer analysis by AMS represented only $0.008 \% \mathrm{w} / \mathrm{w}$ of the total $100 \mathrm{mg}$ acalabrutinib oral dose and therefore did not meaningfully contribute to the area under the concentration-time curve (AUC) of unlabeled acalabrutinib (Sarapa et al., 2005; Lappin and Stevens, 2008).

Cohort 2: Excretion Study. A single dose of $\left[{ }^{14} \mathrm{C}\right]$ acalabrutinib $(100 \mathrm{mg})$ was administered as an oral solution (free base equivalent) containing a microtracer $\left(<10 \mu \mathrm{g} ; \leq 1 \mu \mathrm{Ci}\left[{ }^{14} \mathrm{C}\right]\right.$ acalabrutinib in $100 \mathrm{ml}$ of an approximately $0.009 \mu \mathrm{Ci} / \mathrm{ml}$ Tang orange drink solution; Mondelēz International, Deerfield, IL) in male and female healthy subjects $(n=6)$. The routes and rates of excretion of $\left[{ }^{14} \mathrm{C}\right]-$ acalabrutinib were determined by assessment of concentrations of total ${ }^{14} \mathrm{C}$ radioactivity and of acalabrutinib and its metabolites in whole blood, plasma, and urine, as well as the percentage of recovery of the radioactive dose in urine and feces. Blood samples for total ${ }^{14} \mathrm{C}$ determination in whole blood and plasma and unlabeled acalabrutinib determination in plasma were collected at the following time points: predose; at 15, 30, 45, 60, 75, and 90 minutes; at 2 hours and 2 hours 30 minutes; and at $3,4,5,6,8,12,24,48,72,96,120,144$, and 168 hours postdose. Complete urine collections for unlabeled acalabrutinib and total ${ }^{14} \mathrm{C}$ determination were collected and pooled over the following intervals: -12 to 0 (before dose, up to the last void within 20 minutes before dosing), 0-6, 6-12, and 12-24 hours and at 24-hour intervals through 168 hours postdose. Complete feces collections for total ${ }^{14} \mathrm{C}$ determination were collected and pooled from predose (within 24 hours of dosing; if possible), 0-24 hours postdose, and at 24-hour intervals through 168 hours postdose. Total ${ }^{14} \mathrm{C}$ radioactivity was also determined in peripheral blood mononuclear cells (PBMCs) from blood samples collected at the following time points: predose and 1, 12, 24, 48, and 96 hours postdose. PBMCs were isolated from heparinized whole blood samples on the day of collection using the Ficoll-Paque gradient purification method (GE Healthcare, Uppsala, Sweden) and cryopreserved in $90 \%$ fetal bovine serum plus $10 \%$ dimethylsulfoxide. Genotype analysis was performed for select variants of breast cancer resistance protein [BCRP or ABCG2, 421C $>$ A], CYP3A5 [6986A $>$ G], and glutathione $S$-transferase GSTM1 $[-/-($ null $),-/+,+/+]$. The subjects resided continuously at the study center from check-in until 7 days after administration of $\left[{ }^{14} \mathrm{C}\right]$ acalabrutinib.

\section{Quantitative Bioanalysis of Human Samples}

The analysis of radioactivity was performed by Xceleron Inc. by AMS (Germantown, MD). Freeze-dried feces samples were combusted in a sample oxidizer and the resulting $\mathrm{CO}_{2}$ was trapped and analyzed in a liquid scintillation counter. Whole blood, plasma, PBMCs, and urine were analyzed for ${ }^{14} \mathrm{C}$ total radioactivity using a qualified graphitization and AMS assay. Sodium benzoate was employed as a carbon carrier for PBMC and urine samples.

$\left[{ }^{14} \mathrm{C}\right]$ Acalabrutinib concentrations were determined in urine and plasma samples from cohort 1 using a validated fraction collection, graphitization, and AMS analysis (HPLC+AMS assay). After extraction (plasma only), the samples were separated using an Agilent 1200 HPLC System (Agilent Technologies, Santa Clara, CA), equipped with a UV detector and 96-well plate fraction collector and fitted with an XTerra MS C8 HPLC column $\left(4.6 \times 150 \mathrm{~mm}, 3.5 \mu \mathrm{m}, 55^{\circ} \mathrm{C}\right.$; Waters, Milford, MA), that pumped a $10 \mathrm{mM}$ ammonium bicarbonate in water, $\mathrm{pH}$ 9/acetonitrile mobile phase gradient. The effluent was monitored at an $\lambda$ of $230 \mathrm{~nm}$ to ensure separation of $\left[{ }^{14} \mathrm{C}\right]$ acalabrutinib from other metabolites, which was achieved over a 17-minute total run time.

Plasma and urine samples were analyzed for the unlabeled acalabrutinib parent molecule by validated LC-MS/MS methods against a stable labeled internal standard at Bioanalytical Systems Inc. (West Lafayette, IN) after 
TABLE 1

Mean reversible protein binding of $\left[{ }^{14} \mathrm{Clacalabrutinib}(1,3\right.$, or $10 \mu \mathrm{M})$ and $\mathrm{ACP}$ $5862(1$ or $10 \mu \mathrm{M})$ in mouse, rat, dog, and human plasma and human serum albumin and $\alpha 1$-acid glycoprotein in vitro by ultracentrifugation

\begin{tabular}{lrrrrr}
\hline \multirow{2}{*}{ Species } & \multicolumn{2}{c}{ Acalabrutinib } & & \multicolumn{2}{c}{ ACP-5862 } \\
\cline { 2 - 3 } \cline { 5 - 6 } & Bound & Unbound & & Bound & Unbound \\
\hline & & & $\%$ & & \\
Mouse & 75.4 & 24.6 & & 98.6 & 1.4 \\
Rat & 92.0 & 8.0 & 99.8 & 0.2 \\
Dog & 68.4 & 31.6 & & 94.3 & 5.7 \\
Human & 97.5 & 2.5 & 98.6 & 1.4 \\
HSA & 93.7 & 6.3 & ND & ND \\
AGP & 41.1 & 58.9 & ND & ND \\
\hline
\end{tabular}

AGP, acid glycoprotein; HSA, human serum albumin; ND, not determined.

protein precipitation using acetonitrile. The quantification range for acalabrutinib in lithium heparin plasma was $1-1000 \mathrm{ng} / \mathrm{ml}$, whereas that in urine was 50 $5000 \mathrm{ng} / \mathrm{ml}$. The precision (percent coefficient of variation [CV]) and accuracy (percent bias) observed for acalabrutinib ranged from 1.1 to 3.4 and 0.9 to 2.7 in plasma and from 1.1 to 1.9 and -1.3 to -1.1 in urine, respectively.

\section{PK Analysis}

Relevant PK parameters were determined for plasma $\left[{ }^{14} \mathrm{C}\right]$ acalabrutinib (human ADME cohort 1, intravenous only) and acalabrutinib and for whole blood and plasma for total ${ }^{14} \mathrm{C}$ radioactivity. The analyses were performed at Covance using standard noncompartmental methods with Phoenix WinNonlin software (version 6.4; Certara USA Inc., Princeton, NJ). The AUC parameters were calculated using actual sampling times and the linear trapezoidal rule for increasing concentrations and the logarithmic rule for decreasing concentrations (linear-up, log-down method).

\section{Human Mass Balance}

Radioactive ${ }^{14} \mathrm{C}$ recovery in feces was calculated by summation of the amount excreted in each collection interval during the residential period up to 168 hours postdose. One subject withdrew from the study early. Total recovery of radioactivity for this subject was calculated through 72 hours where data were available. Since $>90 \%$ of total radioactivity was recovered, this subject was not excluded from summary statistics for total recovery.

\section{Human Metabolite Identification}

After analysis of plasma, feces, and urine samples by AMS or by LSC for total radioactive content, appropriate samples were pooled across subjects and time points to give one plasma time-proportional pool (Hamilton et al., 1981), one urine sample and one fecal sample for metabolite profile analysis at Xceleron Inc., and one each for metabolite identification at Covance. Acalabrutinib and its metabolites were extracted twice from plasma using methanol and twice from fecal homogenate using dimethylacetamide; urine was analyzed without extraction. The metabolite profiles in pooled urine and feces were determined using HPLC-UV combined with offline radioactivity monitoring by AMS for detection/ quantification, MS for structure elucidation, and UV for retention time matching between HPLC-UV fractionation for AMS and HPLC-UV-MS systems.

\section{Human Sample Extraction and Metabolite Identification}

The pooled plasma sample was extracted by adding methanol $(3000 \mu \mathrm{l})$ to plasma $(1000 \mu \mathrm{l})$; the mixture was sonicated (10 minutes), vortexed (10 minutes), and centrifuged $\left(3210 \mathrm{~g}, 10\right.$ minutes at $\left.4^{\circ} \mathrm{C}\right)$ and the supernatant was separated. A second extraction was executed in the same way, and the combined supernatants were reduced to dryness under a stream of nitrogen at $60^{\circ} \mathrm{C}$. The extract was then reconstituted in water/methanol $(70: 30 \mathrm{v} / \mathrm{v}$, $500 \mu \mathrm{l}$ ), and the radioactivity measured by AMS and compared against an unextracted sample.

The cross-subject and time-pooled fecal homogenate sample (approximately $500 \mathrm{mg}$ ) was mixed with dimethylacetamide $(1500 \mu \mathrm{l})$, vortexed ( 5 minutes), and centrifuged $\left(3210 \mathrm{~g}, 10\right.$ minutes, $\left.4^{\circ} \mathrm{C}\right)$ and the supernatant was separated. A second extraction was executed in the same way, and the combined supernatants were diluted 10 -fold using water. The undiluted feces extract was analyzed by LSC for radioactivity content to determine the extraction efficiency. Diluted sample extracts were also vortexed and analyzed by HPLC-AMS and HPLC-MS/MS.

The pooled urine sample was directly subjected to chromatographic separation by HPLC-AMS and HPLC-MS/MS analysis.

After extraction (plasma only), the rat, dog, and human samples were separated using a Prominence HPLC System (Shimadzu, Kyoto, Japan), coupled with a Q Exactive mass spectrometer (Thermo Fisher Scientific, Waltham, MA) and fitted with a Waters XTerra MS C8 HPLC column $\left(4.6 \times 150 \mathrm{~mm}, 5 \mu \mathrm{m}, 55^{\circ} \mathrm{C}\right)$, that pumped a $10 \mathrm{mM}$ ammonium bicarbonate in water, $\mathrm{pH}$ 9/acetonitrile mobile phase gradient. Separation was achieved over a 66-minute total run time.

\section{Results}

\section{Reversible Plasma Protein Binding and Red Blood Cell Partitioning}

The stability and nonspecific binding of acalabrutinib and major active circulating metabolite ACP-5862 $\left(\mathrm{BTK} \mathrm{IC}_{50}=5.0 \mathrm{nM}\right.$ in a biochemical kinase assay; see a multidisciplinary review available at https://www. accessdata.fda.gov/drugsatfda_docs/nda/2017/210259Orig1s000MultidisciplineR.pdf) was determined in vitro. Both acalabrutinib and ACP5862 independently demonstrated low nonspecific binding (3\%) to the low-binding polycarbonate tubes and $\geq 95 \%$ stability in mouse, rat, dog, monkey, and human plasma under the assay conditions using LC-MS/MS quantification.

The mean in vitro protein binding ratios of both acalabrutinib $(1,3$, and $10 \mu \mathrm{M})$ and ACP-5862 $(1$ and $10 \mu \mathrm{M})$ were independent of the concentrations tested (Table 1). The plasma protein binding of the acalabrutinib metabolite, ACP-5862, was consistently higher than acalabrutinib across the mouse, rat, dog, and human plasma tested, with higher binding in the rat relative to other species. The free fraction of ACP-5862 in human plasma was 2-fold lower than acalabrutinib. Acalabrutinib was $93.7 \%$ and $41.1 \%$ bound in physiologic concentrations of human serum albumin and $\alpha 1$-acid glycoprotein solutions, respectively.

The mean percent distribution of acalabrutinib (1, 3, and $10 \mu \mathrm{M})$ and ACP-5862 (1 and $10 \mu \mathrm{M})$ to blood cells and the calculated bloodto-plasma ratio were predominantly independent of concentration (Table 2). There was a higher plasma-free fraction and a greater

TABLE 2

Mean blood cell partitioning of acalabrutinib $(1,3$, or $10 \mu \mathrm{M})$ and ACP-5862 $(1$ or $10 \mu \mathrm{M})$ incubated in mouse, rat, dog, monkey, and human whole blood in vitro

\begin{tabular}{lccccc}
\hline \multirow{2}{*}{ Species } & \multicolumn{2}{c}{ Acalabrutinib } & & \multicolumn{2}{c}{ ACP-5862 } \\
\cline { 2 - 3 } \cline { 5 - 6 } \cline { 5 - 6 } & Distribution into Blood Cells & Blood-to-Plasma Ratio & & Distribution into Blood Cells & Blood-to-Plasma Ratio \\
\hline Mouse & $\%$ & & $\%$ & \\
Rat & 56.2 & 1.37 & & 23.3 & 0.87 \\
Dog & 29.6 & 0.87 & & 73.7 & 2.54 \\
Human & 49.6 & 0.06 & & 67.4 & 0.40 \\
\hline
\end{tabular}




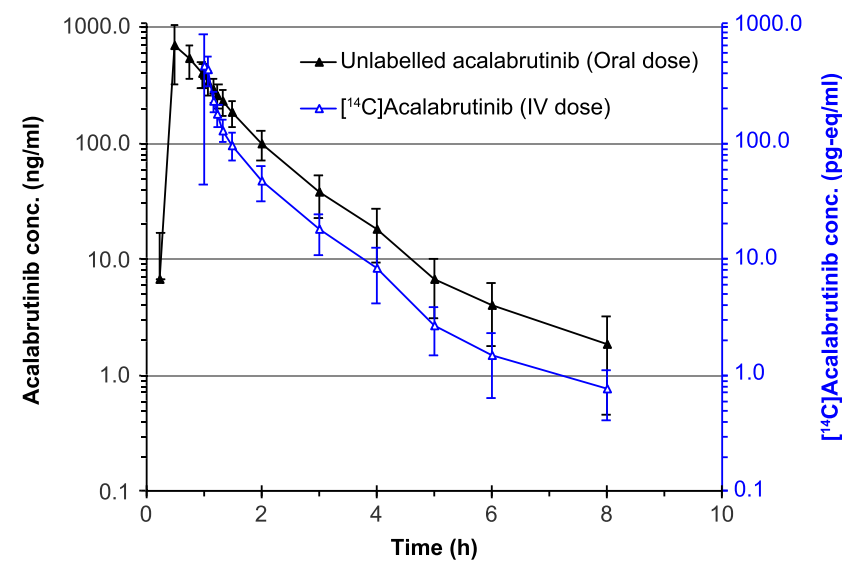

Fig. 2. Arithmetic mean \pm S.D. logarithmic-linear concentration-time profiles for intravenous $\left[{ }^{14} \mathrm{C}\right]$ acalabrutinib and oral unlabeled acalabrutinib in plasma (cohort 1). $\mathrm{IV}$, intravenous.

degree of blood cell partitioning of acalabrutinib in mice and dogs relative to rats and humans.

\section{Human Clinical Study Subject Disposition}

All eight subjects in cohort 1 (three females and five males; mean age \pm S.D. $44 \pm 15.2$ years) and all six subjects in cohort 2 (two females and four males; mean age $38 \pm 17.6$ years) received study medication. All but two subjects (one in each cohort) completed the study. Both subjects withdrew on day 3 for personal reasons, and most PK parameters could be estimated since $>90 \%$ of radioactivity was excreted at the time of withdrawal. There were no reported adverse events that were greater than Common Terminology Criteria for Adverse Events grade 1.

\section{Human Plasma PK (Cohort 1, Absolute Bioavailability)}

The single intravenous dose of $\left[{ }^{14} \mathrm{C}\right]$ acalabrutinib ranged from 7.61 to $7.75 \mu \mathrm{g}$ and the radioactive dose ranged from 961 to $978 \mathrm{nCi}$. Plasma concentration-time curves after administration of a single oral dose of unlabeled acalabrutinib in its $100 \mathrm{mg}$ to-be-marketed capsule formulation and the $\left[{ }^{14} \mathrm{C}\right]$ acalabrutinib microtracer administration 58 minutes later as a single intravenous dose in cohort 1 are shown in Fig. 2, with respective PK parameters summarized in Table 3 . The geometric mean exposure ratio (oral/intravenous) for the acalabrutinib dose-normalized AUC from time 0 to infinity $\left(\mathrm{AUC}_{0-\infty}\right.$; in nanogram equivalent hours per gram), as the measure of absolute bioavailability, was $25.3 \%$ (CV $14.3 \%$, Table 3). Administration of the intravenous microtracer dose was within 30 minutes of the median time of maximum observed plasma concentration $\left(T_{\max }\right)$ for the oral acalabrutinib dose. Oral absorption of acalabrutinib was rapid ( $T_{\max }$ range, $0.5-0.75$ hours), and plasma concentrations of acalabrutinib and $\left[{ }^{14} \mathrm{C}\right]$ acalabrutinib declined in an exponential fashion; this resulted in similar terminal $t_{1 / 2}$ values between oral and intravenous doses, with geometric means of 1.57 (CV 0.60\%) and 1.78 ( $\mathrm{CV} 0.46 \%)$ hours, respectively. After the $\left[{ }^{14} \mathrm{C}\right]$ acalabrutinib intravenous microtracer dose, total body clearance (CL) was moderate at 39.4 1/h (CV 30.6\%) or approximately $45 \%$ of liver blood flow; the volume of distribution was determined to be 98.01 (CV 42.8\%), and the apparent volume of distribution at steady state was 34.21 (CV 40.2\%). Less than $2 \%$ of the dose was excreted in urine after oral and intravenous administration. Renal CL of acalabrutinib after an oral dose (geometric means of $1.21 \mathrm{l} / \mathrm{h}$ and $1.33 \mathrm{l} / \mathrm{h}$ for cohorts 1 and 2 , respectively) accounted for $<1 \%$ of the apparent total clearance (CL/F) of acalabrutinib in both cohorts. Similarly, renal CL of $\left[{ }^{14} \mathrm{C}\right]$ acalabrutinib after an intravenous dose (geometric mean of $0.654 \mathrm{l} / \mathrm{h}$; CV 55.6\%) accounted for $<1 \%$ of the $\mathrm{CL}$ of $\left[{ }^{14} \mathrm{C}\right]$ acalabrutinib. Collectively, these data indicate that a minor amount of parent acalabrutinib was eliminated by the renal route.

\section{Blood and Plasma PK of an Oral $\left[{ }^{14} \mathrm{C}\right]$ Acalabrutinib Dose in Rats, Dogs, and Humans}

Rats. Acalabrutinib represented $11.3 \%$ of the AUC from time 0 to the last quantifiable concentration $\left(\mathrm{AUC}_{0-t}\right.$; in nanogram equivalent hours per gram) for total radioactivity in male and female rat plasma

TABLE 3

Summary of geometric mean PK parameters of acalabrutinib and total ${ }^{14} \mathrm{C}$ radioactivity in healthy human subjects after $100 \mathrm{mg}$ oral and $\leq 10 \mu \mathrm{g}$ intravenous doses Values are presented as geometric means $(\% \mathrm{CV})$ unless otherwise indicated.

\begin{tabular}{|c|c|c|c|c|c|}
\hline \multirow[b]{2}{*}{ Parameter } & \multirow{2}{*}{$\begin{array}{l}\text { Acalabrutinib } \\
\text { Oral Dose Cohort } 1 \\
\quad(n=8)\end{array}$} & \multirow[b]{2}{*}{$\begin{array}{c}{\left[{ }^{14} \mathrm{C}\right] \text { Acalabrutinib }} \\
\text { Intravenous Dose Cohort } 1(n=8)^{c}\end{array}$} & \multirow{2}{*}{$\begin{array}{c}\text { Acalabrutinib } \\
\text { Plasma Cohort } 2 \\
(n=6)\end{array}$} & \multicolumn{2}{|c|}{ Total ${ }^{14} \mathrm{C}^{d}$} \\
\hline & & & & $\begin{array}{c}\text { Plasma Cohort } 2 \\
(n=6)\end{array}$ & $\begin{array}{l}\text { Whole Blood Cohort } 2 \\
\qquad(n=6)\end{array}$ \\
\hline$C_{\max }(\mathrm{ng} / \mathrm{ml})$ & $639(53.8)$ & $510(56.3)$ & $305(44.0)$ & $1340(34.4)$ & $1060(31.3)$ \\
\hline$T_{\max }(\mathrm{h})^{a}$ & $0.50(0.50-0.75)$ & $0.075(0.033-0.117)$ & $0.5(0.5-0.517)$ & $0.875(0.750-2.00)$ & $0.750(0.750-2.00)$ \\
\hline $\mathrm{AUC}_{0-t}(\mathrm{ng} \cdot \mathrm{h} / \mathrm{ml})$ & $643(39.7)$ & $201(32.6)$ & $386(39.2)$ & $5930(29.4)$ & $11,500(27.1)$ \\
\hline $\mathrm{AUC}_{0-12 \mathrm{~h}}(\mathrm{ng} \cdot \mathrm{h} / \mathrm{ml})$ & $642(35.9)$ & $199(30.2)$ & $406(36.6)$ & $4390(29.4)$ & $3760(29.6)$ \\
\hline $\operatorname{AUC}_{0-168 \mathrm{~h}}(\mathrm{ng} \cdot \mathrm{h} / \mathrm{ml})$ & ND & ND & 407 (36.6) & $6250(24.9)$ & $11,500(27.1)$ \\
\hline $\operatorname{AUC}_{0-\infty}(\mathrm{ng} \cdot \mathrm{h} / \mathrm{ml})$ & $643(35.7)$ & $199(30.2)$ & 407 (36.6) & $6410(25.1)$ & ND \\
\hline$t_{1 / 2}(\mathrm{~h})$ & $1.57(0.600)$ & $1.78(0.461)$ & $1.47(0.45)$ & $46.5(21.8)$ & $395(9.6)^{e}$ \\
\hline CL or CL/F (1/h) & $163(35.7)$ & $39.4(30.6)$ & $246(36.6)$ & ND & ND \\
\hline$V_{\mathrm{z}}$ or $V_{\mathrm{z}} / \mathrm{F}(1)$ & $344(36.2)$ & $98.0(42.8)$ & $499(36.1)$ & ND & ND \\
\hline$V_{\mathrm{ss}}(1)$ & ND & $34.2(40.2)$ & ND & ND & ND \\
\hline $\mathrm{F}(\%)^{b}$ & $25.3(14.3)$ & NA & NA & NA & NA \\
\hline Acalabrutinib/total ${ }^{14} \mathrm{C} \mathrm{AUC}_{(0-\infty)}$ ratio & ND & ND & NA & $0.0635(17.8)$ & NA \\
\hline Renal CL $(1 / \mathrm{h})$ & $1.21(32.8)$ & $0.654(55.6)$ & $1.33(36.0)$ & ND & ND \\
\hline Cumulative $\% \mathrm{f}_{\mathrm{eu}}(\%)$ & $0.759(28.3)$ & $1.69(45.7)$ & $0.519(56.5)$ & ND & ND \\
\hline
\end{tabular}

$\% \mathrm{f}_{\text {eu }}$, percent excreted in urine over the entire sample collection period; F, bioavailability fraction of dose absorbed relative to intravenous dosing expressed as a percentage; NA, not applicable; $\mathrm{ND}$, not determined; $V_{\mathrm{ss}}$, volume of distribution at steady state; $V_{\mathrm{z}}$, volume of distribution based on the terminal phase.

${ }^{a}$ Values are the median (minimum-maximum).

${ }^{b}$ Absolute bioavailability was calculated using the dose-normalized $\mathrm{AUC}_{0-\infty}$ after oral and intravenous administration due to differing doses with each route of administration, according to the following equation: $\mathrm{F}=\left[\mathrm{AUC}_{0-\infty}\right.$ (oral)/AUC $\mathrm{AL}_{0-\infty}$ (intravenous) $] /[$ dose (intravenous)/dose (oral) $]$.

${ }^{c}$ Units are $(\mathrm{pg} \mathrm{Eq} / \mathrm{ml})$ or $(\mathrm{pg} \mathrm{Eq} \cdot \mathrm{h} / \mathrm{ml})$.

${ }^{d}$ Units are $(\mathrm{ng} \mathrm{Eq} / \mathrm{ml})$ or $(\mathrm{ng} \mathrm{Eq} \cdot \mathrm{h} / \mathrm{ml})$

$e_{n}=5$. Half-life value is greater than two times the sampling interval. 

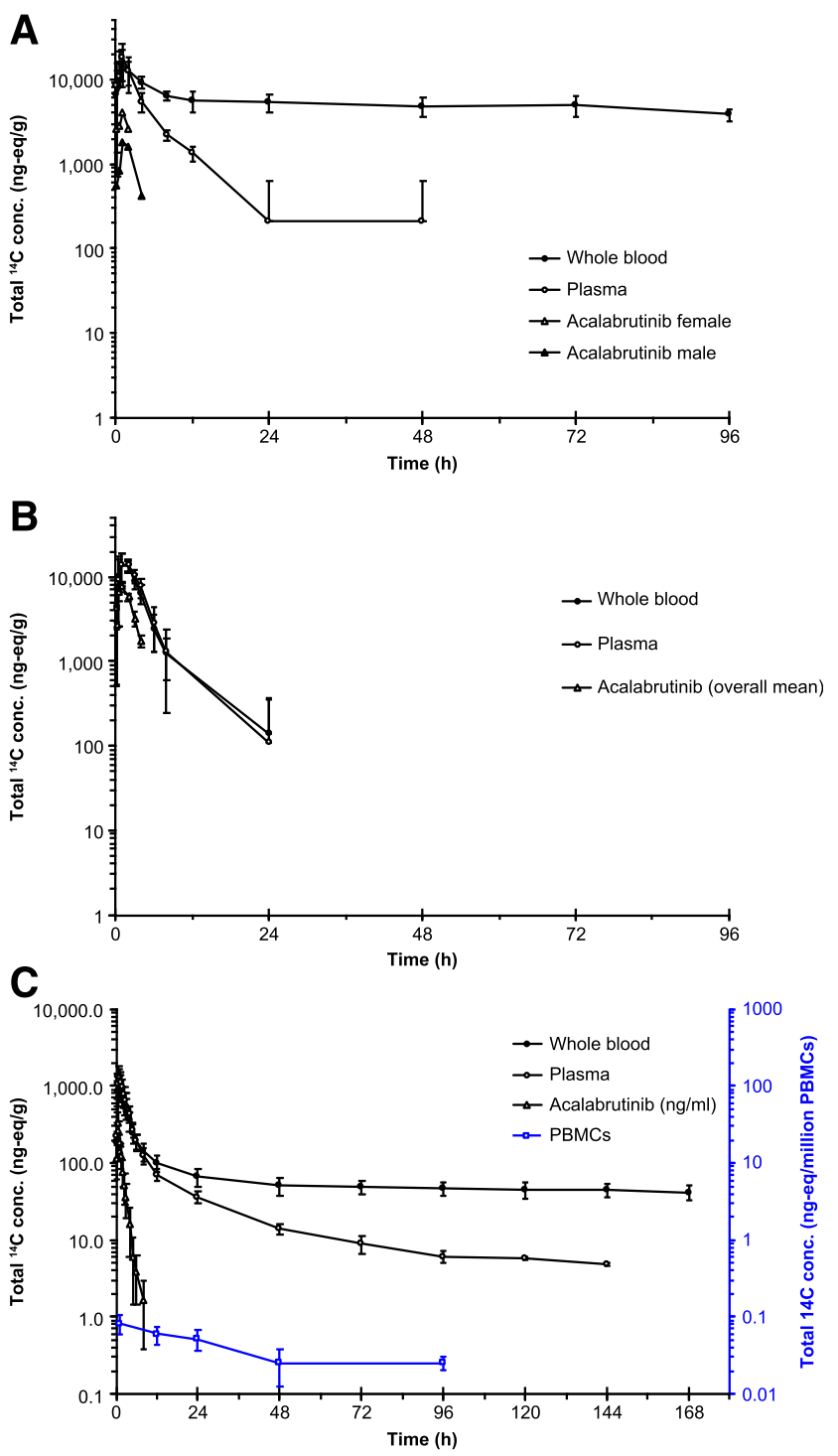

Fig. 3. Arithmetic mean \pm S.D. logarithmic-linear concentration-time profiles for rat total ${ }^{14} \mathrm{C}$ in blood and plasma and acalabrutinib in plasma of male and female rats (A), dog total ${ }^{14} \mathrm{C}$ in blood and plasma and acalabrutinib in plasma of overall dogs $(\mathrm{B})$, and human total ${ }^{14} \mathrm{C}$ in blood, plasma, and PBMCs and acalabrutinib in plasma (cohort 2) (C).

(Supplemental Table 1). The total radioactivity concentrations observed in whole blood and plasma were similar during the initial absorption and rapid elimination phases through 2 hours postdose (Fig. 3). Then a trend toward increased blood-to-plasma concentration ratios over time was observed, ranging from a mean of $0.732-5.31$ for both sexes, which indicated that drug-related radioactivity was increasingly associated with the cellular fraction of blood at later time points. The terminal halflives calculated for blood were long (215 and 157 hours for males and females, respectively). For plasma, $t_{1 / 2}$ values were at least 20 -fold shorter (10.7 and 4.10 hours for males and females, respectively). There was a sex difference with systemic exposure of drug-related radioactivity (approximately $40 \%$ higher for female animals than males).

Dogs. Relative to rats, a lower proportion of the dose was metabolized in dogs, wherein acalabrutinib represented $45.9 \%$ and $41.9 \%$ of the $\mathrm{AUC}_{0-t}$ total radioactivity in male and female dog plasma, respectively (Supplemental Table 2). The plasma and blood total radioactivity concentration versus time profiles were overlapping (Fig. 3). The elimination half-lives for blood and plasma were short for most animals, with measurable radioactivity observed in all animals through 8 hours postdose, and most animals had concentrations below the limits of quantification at 24 hours postdose. Systemic exposure was comparable between blood and plasma and between sexes. Blood/plasma concentration ratios of total radioactivity were consistent from 0.25 to 8 hours postdose and ranged from a mean of $0.813-1.20$ for both sexes, indicating that drug-related radioactivity partitioned freely between the plasma and the cellular fraction of blood.

Humans (Cohort 2, Mass Balance). The single oral dose of acalabrutinib ranged from 108.47 to $108.53 \mathrm{mg}$ and the radioactive dose ranged from 994.53 to $995.06 \mathrm{nCi}$. Plasma concentration-time curves for unlabeled acalabrutinib (LC-MS/MS) and total radioactivity (AMS) are shown in Fig. 3. Like the acalabrutinib parent molecule, total radioactivity in plasma and whole blood revealed rapid absorption after the oral dose ( $T_{\max }$ range, $0.75-2$ hours). Parent acalabrutinib plasma concentrations were a small fraction of the concentrations of total radioactivity, and there were no notable sex- or ADME genotype-related PK differences among the six individual subjects.

Blood and plasma concentrations of total ${ }^{14} \mathrm{C}$ were similar immediately after the dose and for 12 hours postdose. After reaching $C_{\max }$, total ${ }^{14} \mathrm{C}$ concentrations initially declined rapidly, like acalabrutinib. The parent molecule was measurable only up to 8 hours postdose and had a mean elimination half-life $\left(t_{1 / 2}\right)$ value of 1.47 hours. The total ${ }^{14} \mathrm{C}$ in plasma was measurable for 4 days and had a mean terminal $t_{1 / 2}$ value of 46.5 hours (range, 19.6-82.2 hours). At 24 hours postdose and later, the total ${ }^{14} \mathrm{C}$ in whole blood was greater than that observed in plasma and was measurable for the 7-day duration of sample collection. The terminal $t_{1 / 2}$ of these low concentrations of radioactivity in blood ranged from 370 to 465 hours (i.e., the estimated terminal $t_{1 / 2}$ was more than twice as long as the 168-hour sample collection period; $n=5$ ). As in rats, there was also a trend toward an increased blood-to-plasma ratio over time for total radioactivity. The data indicate that small amounts of radioactivity persist in the circulation, with an increasing proportion of the remaining radioactivity attributed to the blood cell fraction, relative to plasma.

After a single oral dose of acalabrutinib/ $\left[{ }^{14} \mathrm{C}\right]$ acalabrutinib, the mean ${ }^{14} \mathrm{C}$ radioactivity per million $\mathrm{PBMCs}$ (the blood cell fraction containing the target BTK) was highest at 1 hour postdose $(0.082 \mathrm{ng} \mathrm{Eq} / \mathrm{million}$ cells) and decreased over time through 48 hours postdose $(0.025 \mathrm{ng}$ $\mathrm{Eq} / \mathrm{million}$ cells).

\section{Excretion and Mass Balance}

Rats. After oral administration of $\left[{ }^{14} \mathrm{C}\right]$ acalabrutinib to male and female Sprague-Dawley rats, the major route of excretion of the administered radioactivity was via the feces (mean $88.3 \%-91.2 \%$; Table 4). Urinary elimination was minor and there was no obvious sex difference in the rates or routes of excretion. Excretion of the administered radioactivity was rapid, with the majority of the dose recovered in the first 48 hours postdose. Concentrations of radioactivity remaining in the individual carcasses at the end of the collection period ( 96 hours postdose) ranged from $0.7 \%$ to $1.0 \%$.

Dogs. After oral administration of $\left[{ }^{14} \mathrm{C}\right]$ acalabrutinib to Beagle dogs, the major route of excretion of the administered radioactivity was via the feces (mean 69.3\%-71.8\%, Table 4). Urinary elimination was lower (mean 14.6\%-15.2\%), and there was no obvious sex difference in the rates or routes of excretion. Excretion of the administered radioactivity was rapid, with most of the dose recovered in the first 48 hours postdose.

Humans (Cohort 2). After a single 100-mg oral dose of acalabrutinib containing a microtracer dose of $\left[{ }^{14} \mathrm{C}\right]$ acalabrutinib, most total ${ }^{14} \mathrm{C}$ radioactivity was eliminated in the feces (Table 4). Geometric mean recoveries of total radioactivity in urine and feces were $12.0 \%$ (minimum-maximum range, $10.3 \%-14.7 \%$ ) and $83.5 \%$ (minimummaximum range, $77.5 \%-86.9 \%$ ), respectively. Approximately $96 \%$ of 
TABLE 4

Summary of the mean total recovery of radioactivity $0-96$ hours after an oral dose of $\left[{ }^{14} \mathrm{C}\right]$ acalabrutinib to male and female rats and dogs and the geometric mean 0-168 hours after an oral dose to male and female human subjects expressed as a percentage of administered dose

Values are presented as means \pm S.D. and geometric means $(\% \mathrm{CV})$.

\begin{tabular}{|c|c|c|c|c|c|}
\hline \multirow{3}{*}{ Type of Sample } & \multicolumn{5}{|c|}{ Administered Dose Recovered } \\
\hline & \multicolumn{2}{|c|}{ Rat } & \multicolumn{2}{|c|}{ Dog } & \multirow{2}{*}{$\frac{\text { Human }}{\text { Overall }(n=6)}$} \\
\hline & Male $(n=3)$ & Female $(n=3)$ & Male $(n=3)$ & Female $(n=3)$ & \\
\hline & \multicolumn{5}{|c|}{$\%$} \\
\hline Urine & $2.7 \pm 0.8$ & $3.6 \pm 0.3$ & $15.2 \pm 1.4$ & $14.6 \pm 3.0$ & $12.0(15.9)$ \\
\hline Feces & $91.2 \pm 6.0$ & $88.3 \pm 3.6$ & $71.8 \pm 0.7$ & $69.3 \pm 7.9$ & $83.5(5.1)$ \\
\hline Cage rinse & $0.2 \pm 0.03$ & $0.8 \pm 0.4$ & $2.5 \pm 0.9$ & $1.6 \pm 1.9$ & NA \\
\hline Carcass & $0.7 \pm 0.1$ & $1.0 \pm 0.5$ & NA & NA & NA \\
\hline Total & $96.8 \pm 3.5$ & $94.3 \pm 2.6$ & $91.1 \pm 0.5$ & $87.5 \pm 6.3$ & 95.7 (4.5) \\
\hline
\end{tabular}

NA, not applicable.

total radioactivity was recovered in the excreta; $>80 \%$ of the total radioactivity was recovered within 96 hours postdose.

\section{Metabolite Profiling}

Across multiple in vitro and in vivo preclinical metabolite profiling studies, more than three dozen acalabrutinib-related peaks were assigned either via high-resolution mass defect filtering or radiochromatographic analysis (data on file). Of these, 15 human and 20 rat and dog metabolite structures were assigned and characterized using a cutoff of $>1 \%$ of dose (urine and feces) or $>1 \%$ of AUC (plasma) for the human ADME study. This was based on ${ }^{14} \mathrm{C}$ analysis of time-proportional pooled samples and a cutoff of $>1 \%$ of total integrated radioactivity in each radiochromatographic analysis (run) for the rat and dog ADME studies, respectively, in plasma, urine, and feces. Three primary metabolic pathways were characterized, with secondary and tertiary metabolites arising from sequential metabolism and/or combinations of the three major routes (Fig. 1). The three primary metabolic pathways were as follows: amide hydrolysis resulting in loss of the 2-aminopyridine group (M1, ACP-5197(4-[8-amino-3-[(2S)-1-but-2-ynoylpyrrolidin-2-yl]imidazo[1,5-a]pyrazin-1-yl]benzoic acid), and M2); GSH or cysteine Michael addition to the 2-butynamide moiety to afford novel enol thioether conjugates M5 (ACP-5530, (2S)-2-amino-5-[[(1R)-1-[[(E)-3[(2S)-2-[8-amino-1-[4-(2-pyridylcarbamoyl)phenyl]imidazo[1,5-a]pyrazin3-yl]pyrrolidin-1-yl]-1-methyl-3-oxo-prop-1-enyl]sulfanylmethyl]2-(carboxymethylamino)-2-oxo-ethyl]amino]-5-oxo-pentanoic acid) and M10 (ACP-5461, (2R)-2-amino-3-[(E)-3-[(2S)-2-[8-amino-1-[4-(2pyridylcarbamoyl)phenyl]imidazo[1,5-a]pyrazin-3-yl]pyrrolidin-1-yl]1-methyl-3-oxo-prop-1-enyl]sulfanyl-propanoic acid), respectively; and pyrrolidine hydroxylation, predominantly at the $\alpha$-methine carbon of the pyrrolidine ring, to afford active, ring-opened, major circulating metabolite, M27 (ACP-5862). An additional biotransformation pathway was conversion of the warhead alkyne to a $\beta$-ketoamide, via hydrolysis of one of the enol thioether conjugates (M23, ACP-5134, 4-[8-amino-3-[(2S)-1(3-oxobutanoyl)pyrrolidin-2-yl]imidazo[1,5-a]pyrazin-1-yl]-N-(2-pyridyl) benzamide; T. Podoll et al., manuscript in preparation).

Rat Plasma. ACP-5862 (M27) was the major single metabolite in the systemic circulation and accounted for $57.4 \%$ and $42.1 \%$ of the $\mathrm{AUC}_{0-t}$ total radioactivity in male and female rat plasma, respectively, which was approximately 4- to 5-fold higher than the parent molecule at $11.3 \%$ (Supplemental Fig. 1; Supplemental Table 1). Four additional metabolites [M1 (amide hydrolysis product), M5, M10, and M23] were also detected in rat plasma (Fig. 1), but with lower $\mathrm{AUC}_{0-t}$ values that ranged from $4.2 \%$ to $12.0 \%$ of the $\mathrm{AUC}_{0-t}$ for total radioactivity in plasma.
Dog Plasma. M1, M5, M7/M40 in aggregate, M10, M25, and M27 were detected in dog plasma (Fig. 1), with $\mathrm{AUC}_{0-t}$ values that ranged from $1.6 \%$ to $21.7 \%$ of the $\mathrm{AUC}_{0-t}$ for total radioactivity in plasma (Supplemental Fig. 2; Supplemental Table 2). ACP-5862 (M27), the major human metabolite, was a relatively minor component in the systemic circulation and accounted for $6.1 \%$ and $8.1 \%$ of the $\mathrm{AUC}_{0-t}$ total radioactivity in male and female dog plasma, respectively. In contrast to rats and humans, the major circulating metabolites in dog plasma are formed by GSH conjugation of the alkyne moiety of acalabrutinib to afford M5 (GSH conjugate, ACP-5530) and sequential cleavage of M5 to the cysteinyl-glycine (M7, ACP-5531, 2-[[(2R)-2amino-3-[(E)-3-[(2S)-2-[8-amino-1-[4-(2-pyridylcarbamoyl)phenyl] imidazo[1,5-a]pyrazin-3-yl]pyrrolidin-1-yl]-1-methyl-3-oxo-prop-1-enyl] sulfanyl-propanoyl]amino]acetic acid) and cysteine conjugates (M10, ACP-5461). Direct chemical reaction of the 2-butynamide warhead with GSH, Cys-Gly, or Cys is a possible alternative to the GSTM1-mediated formation of the GSH conjugate that was demonstrated in vitro (data on file).

Human Plasma. The extraction efficiency of the plasma pools was $85 \%$ and the column fraction recovery was $100 \%$. Profiling of ${ }^{14} \mathrm{C}$ showed that acalabrutinib was extensively metabolized (Fig. 4). In timeproportionally pooled human plasma $(n=6)$, parent acalabrutinib accounted for $8.6 \%$ of total radioactivity (Table 5 ). The most abundant metabolite was M27 (ACP-5862), representing 34.7\% of total radioactivity, which resulted from primary oxidation of the pyrrolidine ring. M27 was the only single human metabolite representing $>10 \%$ of radioactivity and was about 4-fold greater than the amount of parent acalabrutinib in the plasma pool. The next most abundant plasma metabolite components after M27 were 10.8\% (M7, M8, M9, M10, and M11, collectively), 5.9\% (M25), and $2.5 \%$ (M3) of radioactivity in the plasma profile. M7, M9, M10, and M11 represent downstream metabolites of GSH conjugation of the 2-butynamide warhead.

Human Urine. In mass-proportional pooled human urine $(n=6)$, parent acalabrutinib accounted for $0.5 \%$ of the excreted oral dose. The most abundant metabolite cluster coeluted and was $2.7 \%$ of the excreted dose, representing mainly M7, M10, and M11, collectively (Fig. 4). Metabolite M27 (ACP-5862) represented $0.5 \%$ of the excreted dose.

Human Feces. The extraction efficiency of the feces homogenate pools was $89 \%$ and the column fraction recovery was $100 \%$. In massproportional pooled human feces $(n=6)$, parent acalabrutinib accounted for $1.2 \%$ of the excreted dose. The most abundant metabolite cluster coeluted and was $12.1 \%$ of the excreted dose, representing M22, M45, and M23, collectively (Fig. 4). M23 represents a conversion of the acalabrutinib warhead 2-butynamide to a $\beta$-ketoamide (ACP-5134). 

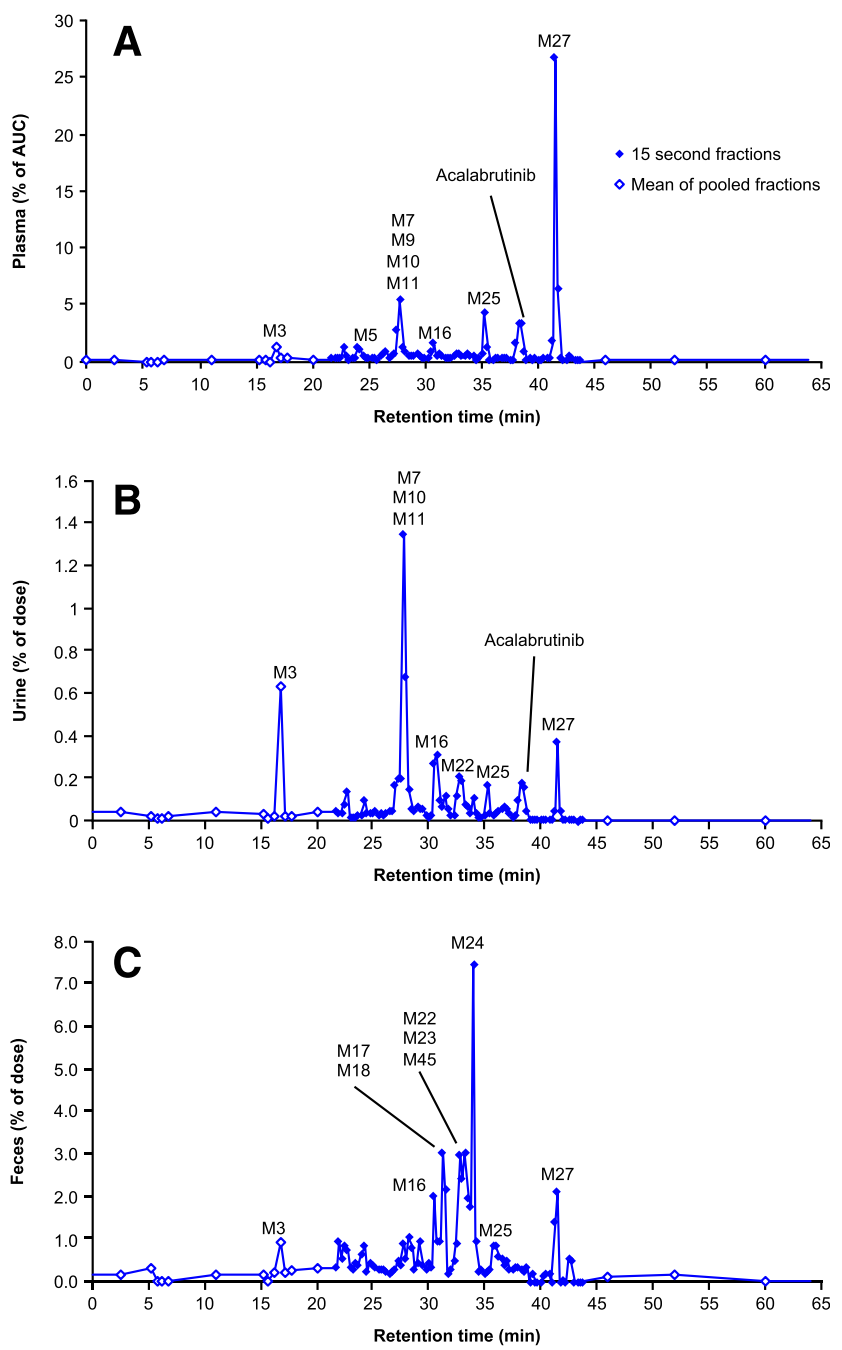

Fig. 4. Reconstructed AMS chromatograms from plasma extract (A), urine (B), and feces extract $(\mathrm{C})$ after a single oral administration of $\left[{ }^{14} \mathrm{C}\right]$ acalabrutinib $(100 \mathrm{mg}, 1 \mu \mathrm{Ci})$ to healthy male and female subjects showing metabolites identified in each matrix.

Empirically, this represents a hydration of the alkyne functional group; however, based on chemical stability, it may be more likely to result from the hydrolysis of enol thioether metabolites in feces (T. Podoll et al., manuscript in preparation). Reduction of the ketone of M23 produced the $\beta$-hydroxybutanamide M45. The next most abundant feces metabolite components were $7.5 \%, 5.2 \%$, and $3.5 \%$ of the excreted dose, representing M24, coeluting M17 and M18, and M27, respectively. M17 and M24 may result from the further oxidation of M45, and M18 and M22 may result from two hydroxylations.

In summary, metabolite profiling and identification data indicate that $\left[{ }^{14} \mathrm{C}\right]$ acalabrutinib underwent extensive metabolism in humans (Fig. 1; Table 5). This occurred primarily by oxidation of its pyrrolidine ring, with one active metabolite, M27 (ACP-5862), appearing in plasma at an exposure that was higher than parent acalabrutinib. Other pathways involved amide hydrolysis, GSH conjugation, and alkyne hydration. The relatively insignificant excretion of parent acalabrutinib in feces and urine indicates that metabolic clearance is the major route of acalabrutinib elimination in humans.

\section{Discussion}

Single oral doses of $100 \mathrm{mg}$ acalabrutinib administered to healthy male and female human subjects in this study were safe and well tolerated. No clinically significant changes or findings were noted from adverse events, clinical laboratory evaluations, vital sign measurements, or 12-lead electrocardiography for this study. Among the male and female subjects enrolled in this study, there was no obvious sex dependence in the PK of acalabrutinib.

In vitro studies established the role of CYP3A and GST (GSTM1 and GSTM2) in the metabolic turnover of acalabrutinib. In addition, acalabrutinib is a substrate for BCRP. Therefore, the effect of mutations resulting in BCRP [421C $>$ A], CYP3A5 [6986A $>$ G], and GSTM1 $[-/-($ null),$-/+,+/+]$ on the disposition of acalabrutinib (Xie et al., 2004; Krivoy et al., 2012; Lee et al., 2015) was investigated. Outcomes from the small number of subjects in cohort 2 of this study, coupled with results from several other healthy subject studies (data on file), indicated that an ADME genotype-related impact on acalabrutinib major metabolic clearance pathways was not likely to occur. Given the predominance of the oxidative metabolic pathway, collectively these data indicate that variability in CYP3A4 expression may be the predominant determinant of acalabrutinib exposure in patients.

The absolute bioavailability of acalabrutinib was established using a microtracer approach, wherein the $\left[{ }^{14} \mathrm{C}\right]$ acalabrutinib intravenous dose was delivered shortly after the $C_{\max }$ for the oral dose of its unlabeled to-be-marketed capsule formulation. Good oral bioavailability $(25 \%)$ was observed. Rapid absorption of the oral dose was followed by rapid elimination with similar $t_{1 / 2}$ values between the oral and intravenous doses. Given the role of CYP3A in the metabolism of acalabrutinib, extraction by gut enzymes during absorption can be anticipated. Concordant with observed bioavailability, acalabrutinib extraction was approximately $50 \%$ for both the gut and the liver (unpublished data) in a $\mathrm{Q}_{\text {gut }}$ model using intravenous metabolic clearance data and CYP3A4 scaling from the gut to the liver (Gertz et al., 2010). By comparison, the first-pass extraction ratio of ibrutinib was estimated to be $92 \%$ with low bioavailability (Scheers et al., 2015). High first-pass metabolism appears to contribute to a higher required daily dose to achieve effective BTK target coverage for ibrutinib at $560 \mathrm{mg}$ daily, compared with the $200 \mathrm{mg}$ total daily dose of acalabrutinib in relapsed/refractory mantle cell lymphoma [see prescribing information for Imbruvica (Pharmacyclics, Sunnyvale, CA) and Calquence].

Acalabrutinib was designed with a less reactive 2-butynamide electrophile to selectively modify cysteine- 481 in the ATP binding pocket of BTK by means of an essentially irreversible covalent bond. Ibrutinib binds irreversibly to BTK via a similar mechanism, employing the more reactive acrylamide electrophile, like other approved covalent kinase inhibitors (afatinib, neratinib, and osimertinib). A previous report established increased kinase selectivity of acalabrutinib relative to ibrutinib and spebrutinib and showed reduced chemical reactivity with GSH in vitro (Barf et al., 2017).

Previous reports of the acrylamide-containing covalent kinase inhibitors have shown significant irreversible binding to off-target proteins (Chandrasekaran et al., 2010; Stopfer et al., 2012; Scheers et al., 2015; Dickinson et al., 2016) that contributed to difficulty measuring the plasma-free fraction in vitro. The reversible plasma protein binding levels of acalabrutinib and ACP-5862 were high and not concentration dependent. The experimental method for noncovalent plasma protein binding studies that was applied to these 2-butynamide covalent inhibitors (acalabrutinib and ACP-5862) indicated recovery and stability consistent with lower binding to off-target proteins than the acrylamide-based TCIs. Acalabrutinib protein binding was lowest in mice and dogs $(75.4 \%$ and $68.4 \%$ bound, respectively), the species with the highest blood cell partitioning (approximately 50\%-56\%). In humans, plasma protein binding averaged 97.5\% and $98.6 \%$ for acalabrutinib and ACP-5862, whereas the blood-toplasma ratio averaged 0.79 and 0.66 , respectively. These data indicate 
TABLE 5

Acalabrutinib metabolites identified in human plasma, urine, and feces

\begin{tabular}{|c|c|c|c|c|c|c|}
\hline \multirow{2}{*}{ Component } & \multirow{2}{*}{ Proposed Identification } & \multirow{2}{*}{ Retention Time } & \multirow{2}{*}{$m / z$} & \multicolumn{2}{|c|}{ Excreted Dose } & \multirow{2}{*}{$\begin{array}{c}\text { AUC } \\
\text { Human Plasma }\end{array}$} \\
\hline & & & & Human Feces & Human Urine & \\
\hline & & $\min$ & \multicolumn{3}{|c|}{$\%$} & \\
\hline M2 & Oxidation, amide hydrolysis & $11.3^{a}$ & 406.1506 & Trace & Trace & ND \\
\hline M3 & Oxidation, dealkylation & $17.2^{a}$ & 431.1459 & 1.8 & 1.3 & 2.5 \\
\hline M37 & {$[\mathrm{M}+\mathrm{H}]^{+}=442$} & $22.1^{a}$ & 442.1983 & Trace & Trace & ND \\
\hline M5 (ACP-5530) & GSH adduct & $24.3^{b}$ & 773.2808 & ND & ND & 2.2 \\
\hline M7 (ACP-5531) & Cysteinylglycine adduct & $27.6^{b}$ & 644.2381 & ND & 2.7 & 10.8 \\
\hline M9 & Oxidized ACP-5531 & $27.7^{b}$ & 660.2328 & ND & & \\
\hline M10 (ACP-5461) & Cysteine adduct & $28.1^{b}$ & 587.2178 & Trace & & \\
\hline M11 & Oxidized ACP-5461 & $28.3^{a}$ & 603.2129 & Trace & & \\
\hline M14 (ACP-5825) & Oxidation (pyrrolidine) & $29.5^{a}$ & 482.1928 & Trace & Trace & ND \\
\hline M16 & Reduction of M27 & $30.9^{a}$ & 484.2088 & 2.9 & 0.6 & 2.2 \\
\hline M17 & $\begin{array}{l}\text { Reduced ACP-5134, pyrrolidine oxidation, } \\
\text { and dehydration }\end{array}$ & $31.6^{a}$ & 484.2087 & 5.2 & Trace & ND \\
\hline M18 & Two oxidations $\left(+\mathrm{O}_{2}\right)$ & $31.4^{c}$ & 498.1879 & & ND & Trace \\
\hline M22 & Two oxidations $\left(+\mathrm{O}_{2}\right)$ & $33.0^{a}$ & 498.1878 & 12.1 & 0.5 & Trace \\
\hline M45 & Reduced ACP-5134 & $33.0^{c}$ & 486.2242 & & Trace & Trace \\
\hline M23 (ACP-5134) & Hydrated alkyne & $33.6^{a}$ & 484.2086 & & Trace & Trace \\
\hline M24 & Reduced ACP-5134 +O & $34.2^{a}$ & 502.2192 & 7.5 & Trace & Trace \\
\hline M25 & Oxidation (pyrrolidine), dehydration & $35.6^{a}$ & 464.1825 & Trace & 0.2 & 5.9 \\
\hline Parent & Acalabrutinib & $38.5^{b}$ & 466.1983 & 1.2 & 0.5 & 8.6 \\
\hline M27 (ACP-5862) & Oxidation (pyrrolidine), ring opening & $41.6^{b}$ & 482.1935 & 3.5 & 0.5 & 34.7 \\
\hline
\end{tabular}

Grouped metabolites coeluted in the same fractions comprising the reconstructed chromatographic trace. Trace indicates that the component was observed via $\mathrm{MS} / \mathrm{MS}$, but ${ }^{14} \mathrm{C}$ was not above baseline. $m / z$, mass-to-charge ratio; ND, not detected.

${ }^{a}$ Retention time from human urine chromatograms.

${ }^{b}$ Retention time from human plasma chromatograms.

${ }^{c}$ Retention times from human feces chromatograms.

that a smaller fraction of the ACP-5862 metabolite total plasma exposure is available for BTK inhibition, relative to the parent molecule. Based on these results, rapid and acceptable recovery of ${ }^{14} \mathrm{C}$ was anticipated during in vivo ADME studies, as little evidence of off-target binding was observed in vitro.

Whole blood and plasma PK for total ${ }^{14} \mathrm{C}$ were very similar in rats, dogs, and humans during the initial absorption phase. They reached similar $C_{\max }$ values and declined rapidly by approximately one log value during the first few hours postdose (Fig. 3). In dogs, parent acalabrutinib was a significant proportion of the total radioactivity, accounting for nearly half of the total ${ }^{14} \mathrm{C} \mathrm{AUC}_{0-t}$ (Supplemental Table 2). In rats and humans, parent acalabrutinib accounted for $11.3 \%$ and $8.7 \%$ of the $\mathrm{AUC}_{0-t}$ for total ${ }^{14} \mathrm{C}$, indicating greater metabolic clearance, relative to dogs. In addition, during the terminal phase of elimination of ${ }^{14} \mathrm{C}$ in rats and humans, a clear trend of an increasing blood-to-plasma ratio was observed as the estimated terminal half-life in the whole blood fraction exceeded the duration of data collection. At the later time points in rats and humans, $>90 \%$ and $>87 \%$ of the administered total radioactivity had been recovered in excreta by 48 and 96 hours postdose, respectively. Measurable amounts of radioactivity persisted in the circulation, an increasing proportion of which was observed in the blood cell fraction relative to plasma. This result may be partly attributed to covalent binding of acalabrutinib to its pharmacological target, BTK, which is expressed in components of whole blood. A visual comparison of the relative concentration of residual radioactivity from acalabrutinib versus ibrutinib in human plasma indicates that covalently bound terminal phase residues may be relatively higher in ibrutinib and could be a manifestation of lower butynamide warhead reactivity in acalabrutinib relative to the reactivity of the acrylamide warhead of ibrutinib (Scheers et al., 2015).

Measurement of target engagement in PBMCs via the ${ }^{14} \mathrm{C}$ label was an exploratory objective of the human ADME study. The mean disappearance of ${ }^{14} \mathrm{C}$ in $\mathrm{PBMCs}$ across the six subjects in cohort 2 was approximately log-linear during the polyexponential elimination from plasma and whole blood (Fig. 3). A portion of the radioactivity observed in the whole blood fraction was confirmed to be associated with PBMCs, the fraction containing the B cells targeted by acalabrutinib therapy. Converting the initial observed ${ }^{14} \mathrm{C}$ concentration in PBMCs to molecules per PBMC resulted in an estimate of approximately 100,000 acalabrutinib molecules per PBMC. It is not clear from these data whether this is a reasonable estimate of BTK content in PBMCs; nonetheless, AMS appears to be a potential (albeit expensive) tool for measuring the elimination of covalently bound BTK from PMBCs or, stated differently, the rate of PBMC BTK resynthesis.

Extrahepatic clearance is a general trait attributed to TCIs based, in part, on the reactivity of the acrylamide electrophile and off-target binding (Shibata and Chiba, 2015; Leung et al., 2017). Given the higher overall $96 \%$ recovery of acalabrutinib-related ${ }^{14} \mathrm{C}$ during the human ADME trial, combined with that specifically observed for extraction of feces samples, irreversible protein binding does not appear to be a meaningful route of extrahepatic clearance for acalabrutinib.

The majority of acalabrutinib metabolism could be summarized by three major routes: oxidation of the pyrrolidine ring, GSH conjugation, and amide hydrolysis. Amide hydrolysis is readily quantifiable in rat and dog plasma (M1, Supplemental Tables 1 and 2) but was below quantifiable limits in human plasma, confirmed instead by the downstream metabolite M2. Although the 2-butynamide warhead is retained in this metabolite, the loss of the 2-pyridylbenzamide likely renders it inactive because of a lack of affinity for the ATP binding pocket (Barf et al., 2017). GSH conjugation of the alkyne moiety of acalabrutinib to afford M5 and sequential cleavage of M5 to the cysteinylglycine (M7) and cysteine conjugates (M10) accounted for a greater proportion of circulating radioactivity in dogs relative to rats and humans. Direct chemical reaction with GSH, Cys-Gly, or Cys with the alkyne warhead is possible, yet a screen for acalabrutinib loss suggested an enzymatic requirement for GSTM1 and GSTM2 (data on file).

By far the most abundant metabolite in the circulation in rats and humans was the oxidation of the pyrrolidine ring resulting in formation 
of the late-eluting, active metabolite M27 (ACP-5862). In vitro BTK inhibition experiments with acalabrutinib and ACP-5862 showed covalent inhibition by both, determined $K_{\mathrm{I}}$ and $k_{\text {inact }}$ values, and demonstrated similar kinase selectivity profiles (T. Podoll et al., manuscript in preparation). The percentage of plasma AUC in humans, as determined by time-proportional pooling, was $34.7 \%$ of the total radioactivity in the circulation, four times greater than parent acalabrutinib (8.6\%). No other metabolites were greater than $10 \%$ of circulating radioactivity. Detailed metabolite structure elucidation studies were conducted to discern the site of oxidation and the final structure of the late-eluting metabolite ACP-5862 (T. Podoll et al., manuscript in preparation).

The $\beta$-ketoamide, M23 (ACP-5134, also a known degradation product of acalabrutinib; data on file), made up a large proportion of the radioactivity excreted in feces (Fig. 4; Table 5). Chemical hydration of the acalabrutinib butynamide warhead in vitro to directly afford ACP-5134 is slow (data on file), and ACP-5134 levels in human plasma are low. The relatively high plasma concentrations of novel enol thioether conjugates (M5, M7, and M10) arising from Michael addition of thiols to the acalabrutinib warhead led to stability studies on the synthetic standard of M5 (ACP-5530). Results showed that enol thioether metabolites are potential precursors of $\beta$-ketoamide-derived metabonates in feces (T. Podoll et al., manuscript in preparation).

Relative to acrylamide-containing TCIs previously approved by the FDA, acalabrutinib is less GSH reactive, and this may account for acalabrutinib's BTK selectivity and lower off-target binding in plasma in this human ADME study. Acalabrutinib's moderate hepatic oxidative metabolic clearance to an active circulating metabolite, coupled with good oral absolute bioavailability that results in high and sustained BTK occupancy in lymphoma patients dosed with a $100 \mathrm{mg}$ twice-daily regimen, all indicate suitable PK for a short half-life covalent agent with extended biologic activity against BTK.

\section{Acknowledgments}

We thank principal investigator Christine Hale (Covance), Brett Pick (Covance) for preparation of the clinical doses, Gina Patel (Covance) for PK analysis, Elizabeth Spencer (Covance) for nonclinical ADME, Bioanalytical Systems Inc. for acalabrutinib clinical sample bioanalysis, Cancer Genetics Inc. for ADME genotyping, Teresa Middleton and Savannah Varney (Covance) for PBMC isolation, Michael Gulrajani and Jean Cheung (Acerta Pharma) for PBMC preparation, and Beverly Stanley, ELS (Team 9 Science LLC), for editorial support.

\section{Authorship Contributions}

Participated in research design: Podoll, Pearson, Evarts, Ingallinera, Bibikova, Slatter.

Conducted experiments: Ingallinera, Sun, Gohdes, Sanghvi.

Performed data analysis: Podoll, Evarts, Ingallinera, Sun, Gohdes, Cardinal, Sanghvi, Slatter.

Wrote or contributed to the writing of the manuscript: Podoll, Pearson, Slatter.

\section{References}

Baillie TA (2016) Targeted covalent inhibitors for drug design. Angew Chem Int Ed Engl 55 : 13408-13421.

Barf T, Covey T, Izumi R, van de Kar B, Gulrajani M, van Lith B, van Hoek M, de Zwart E, Mittag D, Demont D, et al. (2017) Acalabrutinib (ACP-196): a covalent Bruton tyrosine kinase inhibito with a differentiated selectivity and in vivo potency profile. J Pharmacol Exp Ther 363:240-252.

Barf T and Kaptein A (2012) Irreversible protein kinase inhibitors: balancing the benefits and risks. J Med Chem 55:6243-6262.

Buggy JJ and Elias L (2012) Bruton tyrosine kinase (BTK) and its role in B-cell malignancy. In Rev Immunol 31:119-132.

Byrd JC, Harrington B, O'Brien S, Jones JA, Schuh A, Devereux S, Chaves J, Wierda WG, Awan FT, Brown JR, et al. (2016) Acalabrutinib (ACP-196) in relapsed chronic lymphocytic leukemia. $N$ Engl J Med 374:323-332.

Chandrasekaran A, Shen L, Lockhead S, Oganesian A, Wang J, and Scatina J (2010) Reversible covalent binding of neratinib to human serum albumin in vitro. Drug Metab Lett 4:220-227.

Dickinson PA, Cantarini MV, Collier J, Frewer P, Martin S, Pickup K, and Ballard P (2016) Metabolic disposition of osimertinib in rats, dogs, and humans: insights into a drug designed to bind covalently to a cysteine residue of epidermal growth factor receptor. Drug Metab Dispos 44:1201-1212.

Gertz M, Harrison A, Houston JB, and Galetin A (2010) Prediction of human intestinal first-pass metabolism of 25 CYP3A substrates from in vitro clearance and permeability data. Drug Metab Dispos 38:1147-1158.

Hamilton RA, Garnett WR, and Kline BJ (1981) Determination of mean valproic acid serum level by assay of a single pooled sample. Clin Pharmacol Ther 29:408-413.

Krivoy N, Zuckerman T, Elkin H, Froymovich L, Rowe JM, and Efrati E (2012) Pharmacokinetic and pharmacogenetic analysis of oral busulfan in stem cell transplantation: prediction of poor drug metabolism to prevent drug toxicity. Curr Drug Saf 7:211-217.

Lappin G and Stevens L (2008) Biomedical accelerator mass spectrometry: recent applications in metabolism and pharmacokinetics. Expert Opin Drug Metab Toxicol 4:1021-1033.

Lee CA, O'Connor MA, Ritchie TK, Galetin A, Cook JA, Ragueneau-Majlessi I, Ellens H, Feng B, Taub ME, Paine MF, et al. (2015) Breast cancer resistance protein (ABCG2) in clinical pharmacokinetics and drug interactions: practical recommendations for clinical victim and perpetrator drug-drug interaction study design. Drug Metab Dispos 43:490-509.

Leung L, Yang X, Strelevitz TJ, Montgomery J, Brown MF, Zientek MA, Banfield C, Gilbert AM, Thorarensen A, and Dowty ME (2017) Clearance prediction of targeted covalent inhibitors by in vitro-in vivo extrapolation of hepatic and extrahepatic clearance mechanisms. Drug Metab Dispos 45:1-7.

Liu Q, Sabnis Y, Zhao Z, Zhang T, Buhrlage SJ, Jones LH, and Gray NS (2013) Developing irreversible inhibitors of the protein kinase cysteinome. Chem Biol 20:146-159.

Lonsdale R and Ward RA (2018) Structure-based design of targeted covalent inhibitors. Chem Soc $\operatorname{Rev}$ 47:3816-3830.

Moghaddam MF, Tang Y, O'Brien Z, Richardson SJ, Bacolod M, Chaturedi P, Apuy J, and Kulkarni A (2014) A proposed screening paradigm for discovery of covalent inhibitor drugs. Drug Metab Lett 8:19-30.

Ponader S and Burger JA (2014) Bruton's tyrosine kinase: from X-linked agammaglobulinemia toward targeted therapy for B-cell malignancies. J Clin Oncol 32:1830-1839.

Sarapa N, Hsyu P-H, Lappin G, and Garner RC (2005) The application of accelerator mass spectrometry to absolute bioavailability studies in humans: simultaneous administration of an intravenous microdose of ${ }^{14} \mathrm{C}$-nelfinavir mesylate solution and oral nelfinavir to healthy volunteers. J Clin Pharmacol 45:1198-1205.

Scheers E, Leclercq L, de Jong J, Bode N, Bockx M, Laenen A, Cuyckens F, Skee D, Murphy J, Sukbuntherng J, et al. (2015) Absorption, metabolism, and excretion of oral ${ }^{14} \mathrm{C}$ radiolabeled ibrutinib: an open-label, phase I, single-dose study in healthy men. Drug Metab Dispos 43: 289-297.

Shibata Y and Chiba M (2015) The role of extrahepatic metabolism in the pharmacokinetics of the targeted covalent inhibitors afatinib, ibrutinib, and neratinib. Drug Metab Dispos 43:375-384.

Singh J, Petter RC, Baillie TA, and Whitty A (2011) The resurgence of covalent drugs. Nat Rev Drug Discov 10:307-317.

Stopfer P, Marzin K, Narjes H, Gansser D, Shahidi M, Uttereuther-Fischer M, and Ebner T (2012) Afatinib pharmacokinetics and metabolism after oral administration to healthy male volunteers. Cancer Chemother Pharmacol 69:1051-1061.

Wang M, Rule S, Zinzani PL, Goy A, Casasnovas O, Smith SD, Damaj G, Doorduijn J, Lamy T, Morschhauser F, et al. (2018) Acalabrutinib in relapsed or refractory mantle cell lymphoma (ACE-LY-004): a single-arm, multicentre, phase 2 trial. Lancet 391:659-667.

Xie HG, Wood AJ, Kim RB, Stein CM, and Wilkinson GR (2004) Genetic variability in CYP3A5 and its possible consequences. Pharmacogenomics 5:243-272.

Zhao Z and Bourne PE (2018) Progress with covalent small-molecule kinase inhibitors. Drug Discov Today 23:727-735.

Address correspondence to: Dr. Terry Podoll, IV/PO LLC, 5021 Bowen Place South, Seattle, WA 98118-2336. E-mail: terry.podoll@iv-po.com 